\title{
Composite Sequence Dimension
}

National Cancer Institute

\section{Source}

National Cancer Institute. Composite Sequence Dimension. NCI Thesaurus. Code C43438.

Specialized DesignElementDimension to hold CompositeSequences. (caMAGE) 